\title{
Article
}

\section{Mental fatigue impairs physical performance in young swimmers}

Penna, Eduardo, Filho, Edson, Wanner, Samuel, Campos, Bruno, Quinan, Gabriel, Mendes, Thiago, Smith, Mitchell and Prado, Luciano

Available at http://clok.uclan.ac.uk/15608/

Penna, Eduardo, Filho, Edson ORCID: 0000-0002-8548-4651, Wanner, Samuel, Campos, Bruno, Quinan, Gabriel, Mendes, Thiago, Smith, Mitchell and Prado, Luciano (2018) Mental fatigue impairs physical performance in young swimmers. Pediatric Exercise Science, 30 (2). pp. 208-215. ISSN 0899-8493

It is advisable to refer to the publisher's version if you intend to cite from the work. http://dx.doi.org/10.1123/pes.2017-0128

For more information about UCLan's research in this area go to http://www.uclan.ac.uk/researchgroups/ and search for < name of research Group>.

For information about Research generally at UCLan please go to http://www.uclan.ac.uk/research/

All outputs in CLoK are protected by Intellectual Property Rights law, including Copyright law. Copyright, IPR and Moral Rights for the works on this site are retained by the individual authors and/or other copyright owners. Terms and conditions for use of this material are defined in the policies page.

\section{CLoK}

Central Lancashire online Knowledge www.clok.uclan.ac.uk

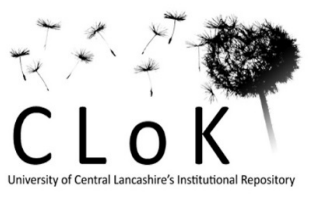


1 Mental fatigue impairs physical performance in young swimmers.

2 Mental fatigue and physical performance in swimmers

3

$4 \quad$ Abstract

5 Purpose: This study aimed to investigate the impact of mental fatigue on heart 6 rate variability $(\mathrm{HRV})$, subjective measures of fatigue, and swimming 7 performance in young athletes. Method: Sixteen swimmers (15.45 \pm 0.51 years 8 old, $7.35 \pm 2.20$ years of swimming experience) performed a $1500-\mathrm{m}$ time trial on 9 two occasions separated by an interval of at least 72 hours. The $1500-\mathrm{m}$ swimming was preceded by a 30-min treatment that consisted of performing the Stroop Color-Word Test to induce mental fatigue (experimental trial), or watching an emotionally neutral video (control trial). Results: Participants reported higher ratings of mental fatigue and mental effort following the Stroop Test when compared to the control trial, but no differences in motivation were observed. The induction of mental fatigue impaired swimming performance, as evidenced by a slower performance (1.2\%) to complete the 1500 -m swimming trial. No inter-trial differences were identified for Rates of Perceived Exertion during the swimming test or in HRV after the Stroop and swimming tests. Conclusion: The results suggest that induction of mental fatigue impairs 1500-m swimming performance without changing HRV.

Key Words: Mental Fatigue, Swimming, Heart Rate Variability 


\section{INTRODUCTION}

Mental fatigue is conceptualized as a psychobiological state induced by sustained periods of demanding cognitive activity and characterized by feelings of tiredness and lack of energy $(6,19)$. The adverse effects of mental fatigue on cognitive performance have been extensively reported $(33,17)$, however, its effects on physical performance have only recently been investigated.

The empirical evidence gathered to date suggests that mental fatigue does not impair short-duration activities requiring all-out strategies (20). However, mental fatigue has been shown to affect athletic performance in longer-duration activities wherein a continuous regulation of effort is necessary. For instance, previous studies have shown the deleterious effect of a mental fatigue state on running $(18,23)$ and cycling performance $(8,19)$. Similarly, an induced state of mental fatigue has been reported to reduce physical and technical performance in football (27), and to impair the accuracy and speed of football-specific decisionmaking (29).

The observed deleterious effects of mental fatigue on physical performance have been primarily attributed to a higher perception of effort in mentally fatigued athletes, as usually assessed by the Rating of Perceived Exertion (RPE) scale (7). It has been suggested that an increased perception of effort could be linked to an augmented activation of the central motor command (i.e., motor-related cortical activity) and its inherent corollary discharges (10). Indeed, when two identical exercises are compared, the individuals subjected to a mental fatigue condition (i.e., experimental condition) show a higher perception of effort compared to a control, despite the absence of other differences in myriad 
50 physiological measures (e.g., heart rate, blood lactate concentrations, oxygen

51 consumption) $(18,19,28)$.

52 Although impaired physical performance during a mental fatigue state appears to occur without concomitant changes in physiological parameters, some studies have revealed that mental fatigue can influence the autonomic regulation of the heart rate $(21,30)$, as evaluated non-invasively through heart rate variability (HRV) analysis. HRV can be defined as over-time variation of consecutive heart beats and is thought to reflect the autonomic nervous system regulation of the heart rate (1). In mental fatigue states, the altered autonomic regulation is characterized by increases in the low-to-high frequency ratio (LF/HF), which indicates that mental fatigue induces sympathetic hyperactivity and decreases parasympathetic activity. In a sporting context, a positive relationship has been identified between rest, increased HF, and improved performance in swimmers $(2,9)$, thus highlighting the importance of verifying possible changes in HRV in mentally fatigued swimmers.

To date, however, no research has examined the effects of mental fatigue in swimmers, who are regularly exposed to long distance training sessions (24) evaluation of a possible influence of mental fatigue on post-exercise HRV is important because sympathetic hyperactivity and parasympathetic underactivity may lead to poor recovery after a training stimulus $(4,22)$. In turn, this imbalance between stimulus and recovery can lead to unhealthy syndromes such as overtraining and burnout (16). 
73 Therefore, the purposes of this investigation were to (a) examine the effects of an induced state of mental fatigue on 1500-m swimming performance, and (b) identify possible alterations in the autonomic control of HR following a prolonged mental exertion task. We hypothesized that mental fatigue would impair swimming performance, while leading to an increased perception of effort and lower values of HRV.

METHODS

\section{Participants}

Sixteen swimmers ( 11 boys and 5 girls, age $15.45 \pm 0.51$ years, $7.35 \pm 2.2$ years of swimming experience) participated in this randomized cross-over investigation. All participants attended school for at least 5 hours per day, were competing in state or national competitions, and trained an average of $30,000 \mathrm{~m}$ per week at the time of the study. Participants and their parents signed an informed consent form outlining potential risks and the study procedures, which were approved by the University's Ethical Advisory Committee (project number 55286716.0.0000.5149).

\section{Experimental Overview}

All participants were instructed to maintain their regular sleep patterns and habitual consumption of caffeine (to avoid a confounding effect due to abstinence). They were also instructed to avoid any vigorous exercise and to take a regular meal at least 24 hours and 2 hours before the two trial sessions. All data collection procedures occurred in the same period of the day and were matched to the athletes' training schedule. The same athlete was always tested at the same time of day. The trial sessions were separated by an interval of at least $72 \mathrm{~h}$. 
97 Upon arrival for the trials, participants received a standard explanation of the procedures, including instructions for the use of the 6-20 RPE scale (7), and were instructed to drink $500 \mathrm{~mL}$ of water. Participants were directed to a quiet room where they completed the visual analogue scales (VAS) for the assessment of mental fatigue $(3,20)$, which was followed by a control or mentally fatiguing treatment. Immediately following the treatment, mental fatigue, mental effort and motivation were assessed using VAS, and heart rate was recorded for 5 min. Participants were then directed to the swimming pool to perform the $1500-\mathrm{m}$ swimming trial. After the swimming trial, the participants were immediately conducted to an isolated, quiet room where they remained seated for 10 minutes. Mental fatigue and mental effort data were gathered and after the initial 5 minutes, their heart rate was recorded. These time intervals were standardized and tightly controlled.

\section{Treatment}

Mental fatigue was induced by a 30-min paper version of a modified Stroop Test. This test has been used in recent studies involving mental fatigue in sporting contexts $(27,28)$. The test required participants to respond verbally to the color of words (red, blue, green and yellow) printed in a random order. The correct answer corresponded to the ink color of the word. For instance, if the ink color of the word was red, the correct answer was the meaning of the word rather than its color. Verbal responses were monitored by a member of the research team, and for each error, the participants were instructed to restart the current line of words. Participants were instructed to respond correctly to as many words as possible for a period of $30 \mathrm{~min}$. 
121 The control trial involved watching a 30-min video regarding the history of world

122 aviation. This video was identified as emotionally neutral (no change in HR, HRV,

123 or mood) in a pilot test.

124 Subjective Ratings

125 To serve as manipulation checks, subjective ratings of mental fatigue, mental 126 effort and motivation were recorded using a 100-mm VAS anchored by the words 127 "not at all" and "maximal"; this scale has been previously used in mental fatigue 128 studies $(27,28)$. Ratings of mental fatigue were measured at pre-treatment (PRE129 TREAT), post-treatment (POST-TREAT) and post-swimming (POST-SWIM). 130 Mental effort was measured at POST-TREAT and POST-SWIM. Motivation was 131 measured only at POST-TREAT and referred to the upcoming 1500-m trial. The 132 VAS was recently used in studies to measure subjective ratings of mental fatigue, 133 mental effort and motivation in sport and exercise context $(19,20,29)$. To analyze 134 the three scales, a ruler was used to measure the distance between the initial mark and the point marked by the participant. Scores were reported as arbitrary units $(A U)$.

$137 \quad H R V$

HRV was measured at two moments (POST-TREAT and POST-SWIM) in both conditions. For all measurements, the participants remained seated for five minutes, with a normal breathing rate, in silence and with no body movements.

To collect the heart rate data, a chest strap (Polar® H7, Kempele, Finland) connected to a recording watch (Polar® V800) was used to continuously record R-R intervals (31). These data were transferred to a Polar software (Polar® ProTrainer) and exported for subsequent analysis using the Kubios HRV version 
1452.0 , which was developed by the Biosignal Analysis and Medical Imaging Group

146 at the Department of Applied Physics, University of Kuopio, Finland.

147 The data were visually inspected to identify ectopic beats and artifacts (which did not exceed $3 \%$ of the recorded data) and those identified were manually removed and replaced by interpolation of their respective adjacent $R-R$ intervals.

To identify the HRV in the time-domain, average $R-R$ intervals ( $R R$ mean) and the root mean square of successive differences between adjacent $R-R$ intervals (RMSSD) were analyzed. A fast Fourier transform of the RR signals was used for analyzing HRV in the frequency-domain. The spectral response provided by the analysis was divided into three bands: very low frequency (VLF; 0.003 to 0.04 $\mathrm{Hz}$ ), low-frequency (LF; $0.04-0.15 \mathrm{~Hz}$ ) and high frequency (HF; 0.15 to $0.40 \mathrm{~Hz}$ ).

\section{Performance Measures}

157 The participants were instructed to swim 1500-m as fast as possible. Data collection was conducted by the same two researchers. One researcher was responsible for recording the pace of each 50-m lap and the time elapsed until finishing the time trial, while the other researcher was responsible for recording the RPE every $300 \mathrm{~m}$. The RPE scale was printed on a $1 \mathrm{~m} \times 0.9 \mathrm{~m}$ banner that was placed beside the pool in a spot perfectly visible to the participants (all trials happened in lanes 1 or 8 ).

Statistical Analysis

Data were initially tested for normality (Shapiro-Wilk test) and homogeneity 166 (Levene test). Because all the data collected passed these two initial tests, parametric tests were performed thereafter. A paired Student $t$-test was 
performed to compare mean data (collected at a single point) between experimental trials. Two-way ANOVAs with repeated measures were used to compare data between experimental trials over distance for different moments (PRE-TREAT, POST-TREAT and POST-SWIM), followed by the Tukey's post hoc test whenever applicable. Additionally, Cohen d magnitude effect-size (ES) was calculated to assess the magnitude of the difference between the experimental trials. ES was calculated through mean differences and was considered trivial (ES < 0.2), small (ES $0.2-0.6$ ), medium (ES $0.6-1.2$ ) and large $(E S \geq 1.2)(13)$. All results are presented as the mean \pm standard deviation. The significance level was set at $p \leq 0.05$. All analyses were performed in the Sigma Plot 11 statistical package.

\section{RESULTS}

\section{Perceptual Measures}

The subjective perception of mental fatigue was influenced by the moment of analysis and experimental condition. Indeed, a two-way ANOVA revealed a significant interaction between these two factors $(F=9.06 ; p<0.001$; power $=$ 0.95). At PRE-TREAT, prior to the Stroop Test or control manipulation, no intertrial differences were observed $(p=0.94 ; E S=0.03)$. As expected, perception of mental fatigue increased after the application of the Stroop Test $(p<0.001$; ES = 2.32), but did not change for the control treatment $(p=0.61 ; E S=0.13)$. Also, the perception of mental fatigue was greater after the Stroop Test than control treatment $(p<0.001$; ES $=1.80)$. In contrast, perception of mental fatigue increased at POST-SWIM relative to POST-TREAT in the control trial $(p<0.01$; $E S=1.34)$, but did not differ following exercise in the mental fatigue trial $(p=$ 0.96; $E S=0.19)($ Figure 1-A) 
193 After the Stroop Test, mental effort was higher than the control treatment $(p<$ 1940.001 ; ES = 2.11). In contrast, mental effort after exercise was not different 195 between trials $(p>0.05 ; E S=0.47)$ (Figure 1-B). When measured at POST196 TREAT, before the swimming time-trail, motivation was not different between 197 trials $(p=0.54 ;$ power $=0.05 ; E S=0.09)$ (Figure $1-C)$.
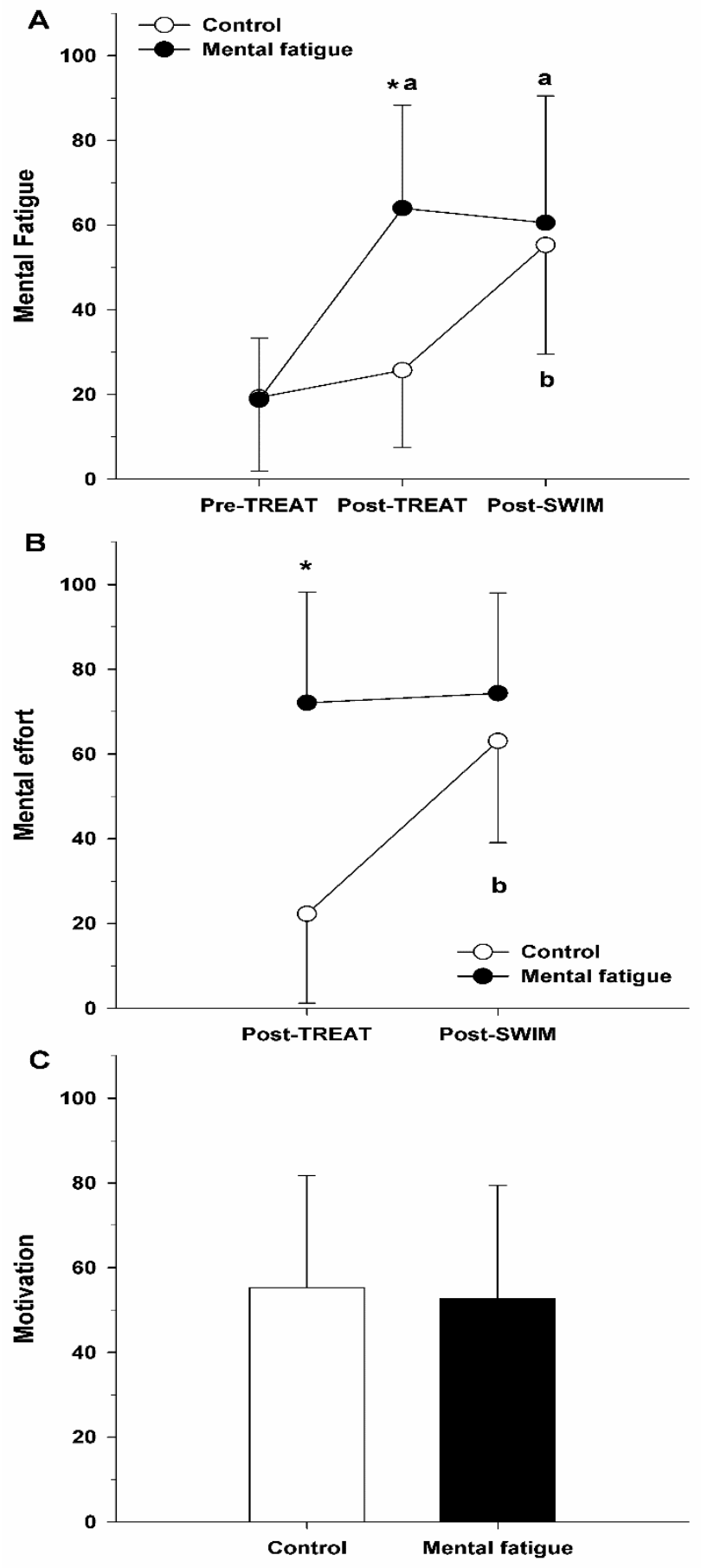
Figure 1. Subjective measure of mental fatigue (A) before treatment (PRE-TREAT), after the Stroop test or control manipulation (POST-TREAT) and at post-swimming (POSTSWIM). Mental effort (B) after the Stroop test or control manipulation (POST-TREAT) and at post-swimming (Post-SWIM). Motivation (C) before the swimming time trial at both trials.

* significantly different $(p<0.05)$ from the control trial; a significantly different $(p<0.05)$ from the previous moment in the mental fatigue trials; b significantly different $(p<0.05)$ from the previous moment in the control trial.

\section{Swimming Performance}

Mental fatigue reduced 1500-m swimming performance, as evidenced by the 1.2 $\pm 1.3 \%$ increase in the time spent to complete the $1500-\mathrm{m}$ time-trial $(p<0.05$ power $=0.70 ; E S=0.13)($ Figure $2 \mathrm{~A})$. Of note, 12 of the 16 swimmers took longer to complete the 1500-m after being subjected to the Stroop Test (Figure 2B). Therefore, mean speed attained by the swimmers was slower in the trial when they were mentally fatigued than during the control trial $(1.169 \pm 0.106 \mathrm{~m} / \mathrm{s} \mathrm{vs}$.

$$
1.155 \pm 0.101 \mathrm{~m} / \mathrm{s} ; p<0.05 ; \text { power }=0.74 ; \mathrm{ES}=0.14) \text {. }
$$
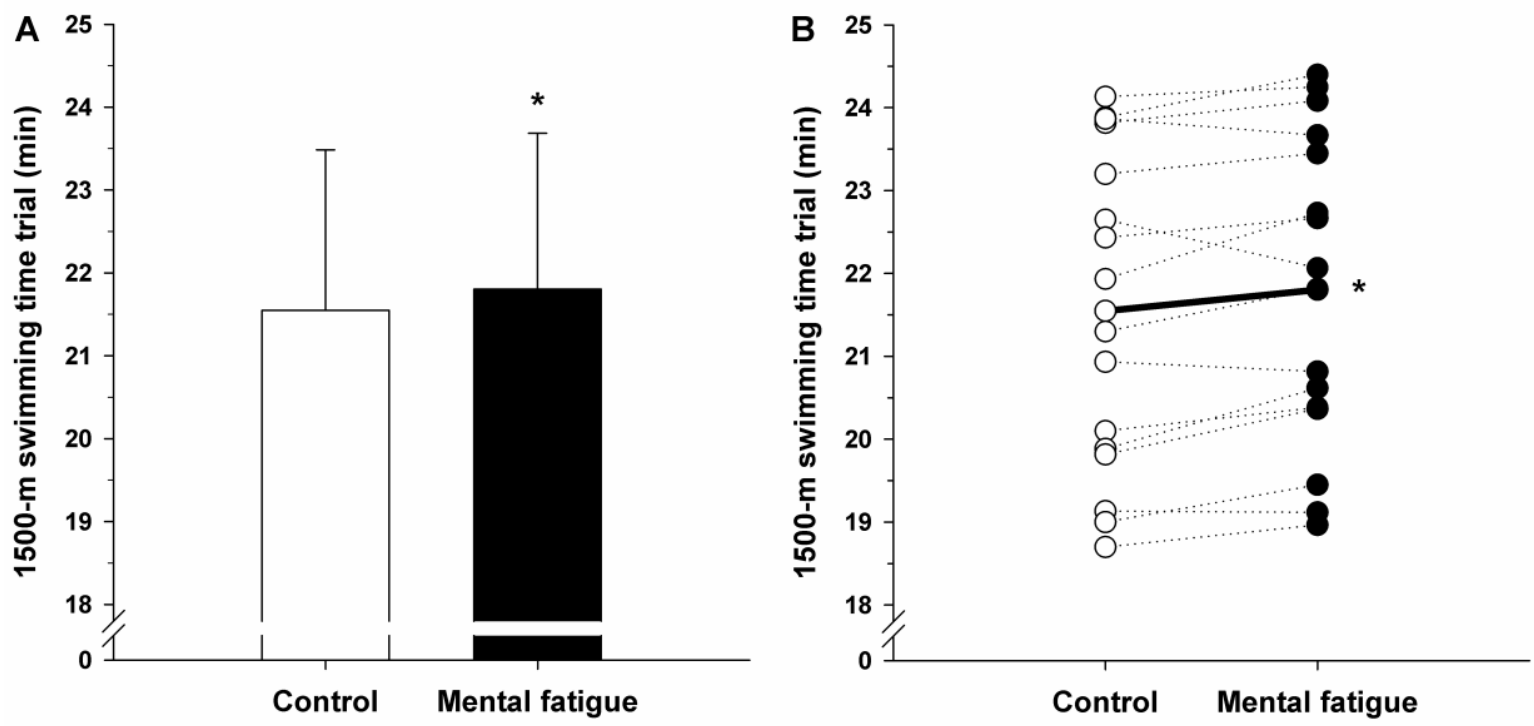

Figure 2. Mean total exercise time (A) and individual times $(B)$ to complete the $1500-\mathrm{m}$ swimming time-trial at the two experimental trials (i.e., control and mental fatigue trials). Each dotted line represents a volunteer, whereas a solid line represents their mean response.

* significantly different $(p<0.05)$ from the control trial. 
222 Swimming pacing profile was influenced by the distance travelled $(F=20.01 ; p<$ 2230.001 ; power $=1.00)$, with swimmers being slower at $600 \mathrm{~m}(\mathrm{p}<0.001$; $E S=$ $2240.39), 900 \mathrm{~m}(\mathrm{p}<0.001 ; \mathrm{ES}=0.30)$ and $1200 \mathrm{~m}(\mathrm{p}=0.01 ; \mathrm{ES}=0.23)$ when compared to $300 \mathrm{~m}$; and being faster at 1500-m when compared to $600 \mathrm{~m}$ (p < $0.001 ; E S=0.32)$ and $900 \mathrm{~m}(p<0.01 ; E S=0.24)$. In addition, pacing mean time

227 for each $300 \mathrm{~m}$ was slower during the mental fatigue trial relative to the control trial $(F=4.62 ; p<0.05 ;$ power $=0.42 ; E S=0.10)$. Regarding the perceptual response, $\mathrm{RPE}$ increased over time $(F=126.25 ; p>0.001 ;$ power $=1.00)$ (Figure

230

231

232

$3-B)$, reaching values close to 20 at the end of the trial. Despite the differences in performance, RPE was not different between experimental trials $(F=0.01 ; p>$ $0.05 ;$ power $=0.05 ; \mathrm{ES}=0.01$ )
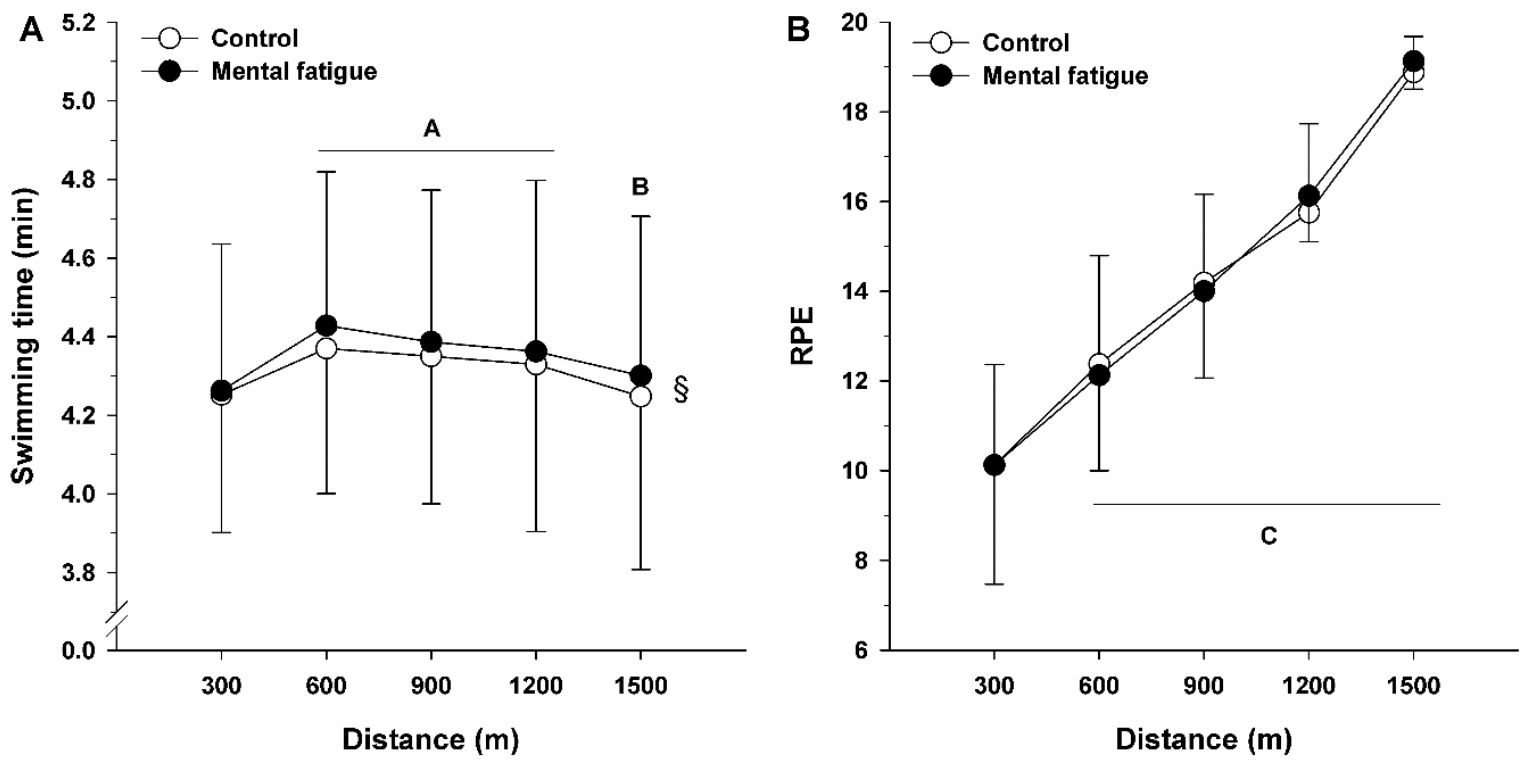

Figure 3. Pacing (A) and rate of perceived exertion (B) during the 1500-m swimming time-trial at both experimental trials. $\S$ significant effect of condition $(p<0.05)$; A significantly different $(p<0.05)$ from the 300 $\mathrm{m}$; B significantly different from the $900 \mathrm{~m}$; C significantly different from the previous distance. 
242 The two-way ANOVA revealed only a main effect for the moment of analysis in

243 the data regarding $R R$ mean $(F=15.49 ; p<0.001$; power $=1.00)$, RMSSD $(F=$ 44.95; $p<0.001 ;$ power $=1.00)$ and LF/HF $(F=9.98 ; p=0.009 ;$ power $=0.79)$. No main effect for experimental trials or interaction between factors were observed. The post hoc tests showed that RR mean $(p<0.001 ; E S=3.98)$ and RMSSD $(p<0.001$; ES = 2.49) were lower at POST-SWIM compared to POSTTREAT, and that LF/HF was higher at POST-SWIM compared to POST-TREAT $(p<0.001 ; E S=1.13)$. No differences in the natural logarithm of low frequency $(F=0.02 ; p=0.91 ;$ power $=0.05 ; E S=0.03), L F(F=0.08 ; p=0.79 ;$ power $=0.05$; $E S=0.06)$, natural logarithm of high frequency $(F=0.11 ; p=0.74 ;$ power $=0.05$; $E S=0.07)$ and HF $(F=0.07 ; p=0.80 ;$ power $=0.05 ; E S=0.05)$ were observed across trials or conditions (Table 1).

\section{*** TABLE 1 HERE ***}

\section{DISCUSSION}

The aim of the present study was to test the hypothesis that a prolonged and demanding cognitive test would lead to a higher perception of mental fatigue state, which in turn would impair swimming performance and alter autonomic cardiac balance. Our findings partially confirmed this hypothesis. Indeed, a prolonged cognitive test was found to increase perception of mental fatigue and impair swimming performance (Figure 2), without concomitant changes in the cardiac autonomic balance of the heart (Table 1). These findings corroborate previous studies that investigated the relationship between mental fatigue status and physical performance $(8,18,28)$. Specifically, previous work in this area has revealed that mental fatigue impairs running performance $(18,27)$. However, to 
268 the best of our knowledge, this is the first study assessing the effects of a mental 269 fatigue on swimming performance.

Noteworthy, the statically significant effect of mental fatigue manipulation on swimming performance (i.e., $0.2 \pm 1.3 \%$ increase in mean time to complete a 1,500-m time-trial) was trivial for both swimming time $(E S=0.13)$ and mean speed $273(E S=0.14)$. However, 12 of the 16 swimmers $(75 \%)$ reduced their physical performance after the mental fatigue manipulation and a delta of $1.2 \%$ in the total exercise time is greater, for example, than the differences found between the three medalists in the 2016 Olympic Games 1500-m swimming (delta of $0.72 \%$ in time to complete the $1500-\mathrm{m}$ of swimming). Thus, the small effects of mental fatigue on physical performance and mean speed could be relevant for a competitive environment.

Even though mental fatigue impaired physical performance, as evidenced by reduced swimming speed (Figure 2), no changes in RPE were observed throughout the 1500-m swimming test (Figure 3). This aligns with the results of other studies $(18,28)$ who observed no between-condition differences in RPE during freely-paced running protocols. Collectively, these results suggest that mental fatigue increases perceived exertion during both fixed and freely-paced endurance exercise. Indeed, during fixed-pace exercise, athletes report higher RPE, whereas during freely-paced exercise athletes regulate their pace to maintain similar RPE between conditions.

It has been suggested that changes in motivation status, due to mental fatigue, may influence physical performance. However, we did not find this to be the case, considering that no inter-trial differences were identified in motivation levels prior to swimming (Figure 1C). Thus, the impaired physical performance after the 
293

294

295

296

297

298

299

300

301

302

303

304

305

306

307

application of the Stroop Test cannot be explained by changes in motivation. In fact, it was shown that mental fatigue is not always associated with task disengagement (12) or reduced motivation (20). Mental fatigue has been rather associated with decreases in other components of cognitive performance, such as cognitive efficiency (measured by impaired reaction time in a prolonged flanker test) $(5,17)$ or availability of cognitive resources $(15)$. Of note, the influence of the aforementioned components of cognition on physical performance are currently unknown.

The perception of mental effort increased in the control trial, while remained high in the mental fatigue trial (Figure 1B). Collectively, these results reflect the fact that long-distance swimming was perceived as mentally effortful (in both trials), and this effort may be associated with the continuous conscious decision-making regarding the regulation of exercise intensity (25). This result aligns with the premise that when a participant engages in long-duration and/or high intensity exercise, their attentional focus remains internal (associative) (14), as particularly related to the regulation of bodily sensations and pacing strategy (32).

The induction of higher perceptions of mental fatigue did not change any HRV parameter investigated in the present study (Table 1). Thus, the hypothesis that mental fatigue reduces the vagal activity and promotes sympathetic hyperactivity was not confirmed. As such, changes in HRV cannot be attributed to any acute physical impairment caused by mental fatigue. This result contrasts with those previously reported $(21,30)$, whose studies showed reduced HRV and increase in sympathetic markers (e.g. Low frequency component) due to mental fatigue. These conflicting findings may have occurred because we examined HRV after the cognitive test, whereas $(21,30)$ examined $H R V$ during the cognitive test. This 
318 methodological difference is important as the predominance of vagal tone could

319 be quickly recovered after the termination of the cognitive task (26), thus not being captured after the conclusion of the physical effort.

\section{PRACTICAL APPLICATIONS}

Previous studies have shown that mental fatigue impairs physical performance in

a variety of sporting contexts. This investigation extends these findings to the context of long distance (1500-m) swimming. The findings of this study are important for coaches and professionals who are responsible for the planning and executing of training programs, particularly those involving young, school-aged athletes. Youth athletes are engaged, on a daily basis, in extensive cognitive tasks (e.g., school) in addition to their training and competition routine. Coaches should be conscious of the impact that these demanding cognitive tasks may have on performance during training sessions. For example, compared to a regular week of class, mental fatigue may be higher during an exam week at school, thus negatively impacting athletic performance. Another important question regarding the swimming context in general and mental fatigue in particular, pertains to the culture of early morning training sessions. Chronic reduced sleep time can negatively influence performance in both cognitive and motor tasks (11). Furthermore, leisure activities involving virtual environments (e.g., electronic games, social media) are very popular among school age populations. These activities may potentially induce mental fatigue.

CONCLUSION

The present data demonstrates that induction of mental fatigue slightly impaired physical performance in young swimmers. Notably, during the mental fatigue 
342 trials, the young athletes presented a similar RPE, but swam at a slower pace

343 than in the control trial. No changes in HRV were observed between conditions.

\section{REFERENCES}

346

347

1. Acharya UR, Joseph ÆEKP, Choo NKÆ, Lim M, Suri ÆJJS. Heart rate variability : a review. Med Biol Eng Comput. 2006;44(1):1031-1051. doi:10.1007/s11517-006-0119-0.

2. Atlaoui D, Pichot V, Lacoste L, Barale F, Lacour JR, Chatard JC. Heart rate variability, training variation and performance in elite swimmers. Int J Sports Med. 2007;28(5):394-400. doi:10.1055/s-2006-924490.

3. Badin O, Smith MR, Conte D, Coutts AJ. Mental Fatigue Impairs Technical Performance in Small-Sided Soccer Games. Int J Sports Physiol Perform. 2016;11(8):1100-1105. doi: 10.1123/ijspp.2015-0710.

4. Baumert M, Brechtel L, Lock J, et al. Heart Rate Variability, Blood Pressure Variability, and Baroreflex Sensitivity in Overtrained Athletes. Clin J Sport Med. 2006;16:412-417. doi:10.1097/01.jsm.0000244610.34594.07.

5. Boksem MAS, Meijman TF, Lorist MM. Effects of mental fatigue on attention : An ERP study. Cogn Brain Res. 2005;25:107-116. doi:10.1016/j.cogbrainres.2005.04.011.

6. Boksem MAS, Tops M. Mental fatigue: Costs and benefits. Brain Res Rev. 2008;59(1):125-139. doi:10.1016/j.brainresrev.2008.07.001.

7. Borg G. Psychophysical bases of perceived exertion. Med Sci Sports Exerc. 1982;14(5):377-381. doi:10.1249/00005768-198205000-00012. 
8. Brownsberger J, Edwards A, Crowther R, Cottrell D. Impact of mental fatigue on self-paced exercise. Int J Sports Med. 2013;34(12):1029-1036. doi:10.1055/s-0033-1343402.

9. Chalencon S, Busso T, Lacour J-R, et al. A Model for the Training Effects in Swimming Demonstrates a Strong Relationship between Parasympathetic Activity , Performance and Index of Fatigue. PLoS One. 2012;7(12):1-10. doi:10.1371/journal.pone.0052636.

10. Cutsem J Van, Marcora S, Pauw K De, Bailey S, Meeusen R, Roelands B. The Effects of Mental Fatigue on Physical Performance : A Systematic Review. Sport Med. 2017;47(8):1569-1588. doi:10.1007/s40279-016-0672-0.

11. Fullagar HHK, Skorski S, Duffield R, Hammes D, Coutts AJ, Meyer T. Sleep and Athletic Performance: The Effects of Sleep Loss on Exercise Performance, and Physiological and Cognitive Responses to Exercise. Sport Med. 2015;45(2):161-186. doi:10.1007/s40279-014-0260-0.

12. Gergelyfi M, Jacob B, Olivier E, Zénon A. Dissociation between mental fatigue and motivational state during prolonged mental activity. Front Behav Neurosci. 2015;9(13):1-15. doi:10.3389/fnbeh.2015.00176.

13. Hopkins WG, Marshall SW, Batterham AM, Hanin J. Progressive statistics for studies in sports medicine and exercise science. Med Sci Sports Exerc. 2009;41(1):3-12. doi:10.1249/MSS.0b013e31818cb278.

14. Hutchinson JC, Tenenbaum G. Attention focus during physical effort: The mediating role of task intensity. Psychol Sport Exerc. 2007;8(2):233-245. doi:10.1016/j.psychsport.2006.03.006. 
15. Kato $\mathrm{Y}$, Endo H, Kizuka T. Mental fatigue and impaired response processes : Event-related brain potentials in a Go / NoGo task. Int J Psychophysiol. 2009;72(2):204-211. doi:10.1016/j.ijpsycho.2008.12.008.

16. Kenttä G, Hassmén P. Overtraining and Recovery: A Conceptual Model. Sport Med. 1998;26(1):1-16.

17. Lorist MM, Boksem MAS, Ridderinkhof KR. Impaired cognitive control and reduced cingulate activity during mental fatigue. Cogn Brain Res. 2005;24(2):199-205. doi:10.1016/j.cogbrainres.2005.01.018.

18. MacMahon C, Schücker L, Hagemann N, Strauss B. Cognitive fatigue effects on physical performance during running. J Sport Exerc Psychol. 2014;36(4):375-381. doi:10.1123/jsep.2013-0249.

19. Marcora SM, Staiano W, Manning V. Mental fatigue impairs physical performance in humans. J Appl Physiol. 2009;106(3):857-864. doi:10.1152/japplphysiol.91324.2008.

20. Martin K, Thompson KG, Keegan R, Ball N, Rattray B. Mental fatigue does not affect maximal anaerobic exercise performance. Eur J Appl Physiol. 2015;115(4):715-725. doi:10.1007/s00421-014-3052-1.

21. Mizuno K, Tanaka M, Yamaguti K, Kajimoto O, Kuratsune H, Watanabe Y. Mental fatigue caused by prolonged cognitive load associated with sympathetic hyperactivity. Behav Brain Funct. 2011;7(1):17. doi:10.1186/1744-9081-7-17.

22. Nummela A, Hynynen E, Kaikkonen P, Rusko H. High-intensity endurance training increases nocturnal heart rate variability in sedentary participants. Biol Sport. 2016;33(1):7-13. doi:10.5604/20831862.1180171. 
412

413

414

415

416

417

418

419

420

421

422

423

424

425

426

427

428

429

430

431

432

433

434

435

23. Pageaux B, Lepers R, Dietz KC, Marcora SM. Response inhibition impairs subsequent self-paced endurance performance. Eur J Appl Physiol. 2014;114(5):1095-1105. doi:10.1007/s00421-014-2838-5.

24. Raglin J, Sawamura S, Alexiou S, Hassmen P, Kentta G. Training Practices and Staleness in 13-1 8-Year-Old Swimmers: A Cross-cultural Study. Pediatr Exerc Sci. 2000;12:61-70.

25. Renfree A, Martin L, Micklewright D, St Clair Gibson A. Application of decision-making theory to the regulation of muscular work rate during selfpaced competitive endurance activity. Sport Med. 2014;44(2):147-158. doi:10.1007/s40279-013-0107-0.

26. Saul JP. Beat-to-Beat Variations of Heart-Rate Reflect Modulation of Cardiac Autonomic Outflow. News Physiol Sci. 1990;5(1):32-37.

27. Smith MR, Coutts AJ, Merlini M, Deprez D, Lenoir M, Marcora SM. Mental fatigue impairs soccer-specific physical and technical performance. Med Sci Sports Exerc. 2016;48(2):267-276. doi:10.1249/MSS.0000000000000762.

28. Smith MR, Marcora SM, Coutts AJ. Mental fatigue impairs intermittent running performance. Med Sci Sports Exerc. 2015;47(8):1682-1690. doi:10.1249/MSS.0000000000000592.

29. Smith MR, Zeuwts L, Lenoir M, Hens N, De Jong LMS, Coutts AJ. Mental fatigue impairs soccer-specific decision-making skill. J Sports Sci. 2016;34(14):1297-1304. doi:10.1080/02640414.2016.1156241.

30. Tanaka M, Mizuno K, Tajima S, Sasabe T, Watanabe Y. Central nervous system fatigue alters autonomic nerve activity. Life Sci. 2009;84(7-8):235239. doi:10.1016/j.Ifs.2008.12.004. 
436 31. Task F. Guidelines: Heart rate variability Standards of measurement, 437 physiological interpretation, and clinical use. Task Force of The European 438 Society of Cardiology and The North American Society of Pacing and $439 \quad$ Electrophysiology. Eur Heart J. 1996;17:354-381.

440 doi:10.1161/01.CIR.93.5.1043.

441 32. Tenenbaum G, Connolly CT. Attention allocation under varied workload and 442 effort perception in rowers. Psychol Sport Exerc. 2008;9(5):704-717. 443 doi:10.1016/j.psychsport.2007.09.002.

444 33. Van der Linden D, Frese M, Meijman TF. Mental fatigue and the control of 445 cognitive processes: Effects on perseveration and planning. Acta Psychol 446 (Amst). 2003;113(1):45-65. doi:10.1016/S0001-6918(02)00150-6. 
458 TABLE 1 - Heart rate variability parameters calculated before and after the 1,500

$459 \mathrm{~m}$ - swimming in the two experimental conditions (mental fatigue and control).

\begin{tabular}{ccccc}
\hline \multicolumn{4}{c}{ Mental fatigue } & \multicolumn{2}{c}{ Control } \\
& POST-TREAT & POST-SWIM & POST-TREAT & POST-SWIM \\
\hline $\begin{array}{c}\text { RR } \\
\text { (ms) }\end{array}$ & $772.4 \pm 99.8$ & $529.5 \pm 46.7$ & $783.7 \pm 79.8$ & $522.2 \pm 28.4$ \\
$\begin{array}{c}\text { RMSSD } \\
\text { (ms) }\end{array}$ & $50.7 \pm 23.1$ & $13.5 \pm 10.0$ & $46.3 \pm 14.3$ & $14.6 \pm 9.6$ \\
$\begin{array}{c}\text { InLF } \\
\text { (ms2) }\end{array}$ & $-2.59 \pm 0.24$ & $-2.63 \pm 0.32$ & $-2.65 \pm 0.33$ & $-2.59 \pm 0.28$ \\
$\begin{array}{c}\text { InHF } \\
\text { (ms2) }\end{array}$ & $-1.66 \pm 0.19$ & $-1.61 \pm 0.32$ & $-1.61 \pm 0.18$ & $-1.62 \pm 0.32$ \\
$\begin{array}{c}\text { LF } \\
\text { (n.u.) }\end{array}$ & $74.7 \pm 12.5$ & $81.0 \pm 16.3$ & $71.9 \pm 13.1$ & $82.3 \pm 11.2$ \\
$\begin{array}{c}\text { HF } \\
\text { (n.u.) }\end{array}$ & $25.1 \pm 12.4$ & $18.8 \pm 16.2$ & $27.9 \pm 13.1$ & $17.4 \pm 11.1$ \\
LF/HF & $3.8 \pm 1.9$ & $8.4 \pm 6.3$ & $3.3 \pm 1.8$ & $6.7 \pm 3.6$ \\
\hline Caption: & mHF & & & \\
\hline
\end{tabular}

461 Caption: InHF $=$ Natural Logarithms of High Frequency; InLF $=$ Natural 462 Logarithms of Low Frequency; HF = High Frequency; LF = Low Frequency; $463 \mathrm{LF} / \mathrm{HF}=$ Ratio; POST-SWIM = Post Swimming; POST-TREAT = Post Treatment; $464 \mathrm{RMSSD}=$ Square root of the mean of the sum of the squares of differences; RR $465=\mathrm{R}-\mathrm{R}$ intervals 